\title{
CALIDAD DE VIDA LABORAL Y EL COMPROMISO ORGANIZACIONAL: UNA PERSPECTIVA DESDE LAS UNIVERSIDADES.
}

Nory Analidhia Pinela Morán, Mgs. Magíster en Dirección de Empresas (Ecuador). Docente Tiempo Completo de la Facultad de Ciencias Económicas y Directora de Posgrado y Educación Continua de la Universidad ECOTEC, Ecuador. Presidenta de PINELA S.A. Ingeniería \& Construcción, Ecuador. npinela@ecotec.edu.ec

Dra. Zoraima Aurelia Donawa Torres Doctora en Ciencias Gerenciales (Venezuela). Docente Asociado e investigadora del Instituto Universitario de Tecnología de Cabimas, estado Zulia, Venezuela. zdonawa62@hotmail.com

\section{ARTÍCULO DE REVISIÓN BIBLIOGRÁFICA}

Recibido: 5 de julio de 2019.

Aceptado: 12 de septiembre de 2019.

\section{RESUMEN}

El objetivo fundamental del presente artículo es determinar la importancia de la calidad de vida laboral y su efecto sobre el compromiso organizacional desde una perspectiva teórica en contextos universitarios. Para ello, se recurre a la revisión bibliográfica que permita perfilar una amplia fundamentación teórica con nuevos enfoques que descomponen las variables calidad de vida laboral y compromiso organizacional identificando los indicadores de comportamiento actitudinal en los docentes de las universidades que permitirá a futuras investigaciones elaborar un instrumento válido y confiable para obtener un diagnóstico de la percepción de las dimensiones de las variables y de su relación, de tal manera que pueda ser utilizado por las organizaciones. Los resultados de la revisión teórica enuncian una relación significativamente positiva entre los componentes de la calidad de vida laboral sobre los componentes del compromiso organizacional.

Palabras clave: calidad de vida laboral, compromiso organizacional, productividad, instituciones de educación superior.

\section{ABSTRACT}


The fundamental objective of this article is to determine the importance of the quality of working life and its effect on organizational commitment from a theoretical perspective in university contexts. To do so, we resort to a bibliographic review that allows us to outline a broad theoretical foundation with new approaches that break down the variables quality of work life and organizational commitment by identifying indicators of attitudinal behavior in university teachers that will allow future research to develop an instrument valid and reliable to obtain a diagnosis of the perception of the dimensions of the variables and their relationship, in such a way that it can be used by organizations. The results of the theoretical review enunciate a significantly positive relationship between the components of the quality of work life on the components of organizational commitment.

Keywords: quality of work life, organizational commitment, productivity, higher education institutions.

\section{INTRODUCCIÓN}

Los constantes cambios que las organizaciones deben enfrentar obligan a los gerentes a implementar nuevas gestiones para incrementar la productividad y alcanzar altos niveles de competitividad. Como parte de esta tendencia surge con mayor fuerza la importancia que en las nuevas formas de gestión empresarial adquieren los estudios sobre el tema de calidad de vida laboral, por ello se han realizado amplias investigaciones en las que se concluye que la calidad de vida laboral debiera ser uno de los elementos más importantes dentro de gerencia de las organizaciones. El concepto de calidad de vida laboral tiene que ver con la satisfacción, la salud y el bienestar del trabajador; así como con todo lo relacionado con su entorno laboral; pero además contiene una perspectiva psicológica con aspectos subjetivos en cada persona, por lo que cada empleado en su ambiente de trabajo determinará en gran medida su calidad de vida laboral (Cruz, 2018). En este sentido, la verdadera utilidad del concepto de calidad de vida laboral, se percibe sobre todo en los servicios humanos, puede ser utilizado para una serie de propósitos, incluyendo la evaluación de las necesidades de las personas y sus niveles de satisfacción (Delgado, López y Moreno, 2017).

Las investigaciones han demostrado la importancia de la calidad de vida laboral en las organizaciones, estableciendo que puede tener un impacto significativo en las respuestas de comportamiento de los empleados, como por ejemplo la disminución en el desempeño para el cumplimiento de las actividades (Matabanchoy, Álvarez y Riobamba, 2019), el ausentismo laboral por situaciones que influyen en el lugar de trabajo como la insatisfacción laboral, donde los trabajadores perciben disminuidas sus posibilidades de expansión y crecimiento profesional, observando condiciones desfavorables e inadecuadas relaciones con los compañeros de trabajo (Tatamuez- Tarapues, Domínguez y Matabanchoy-Tulcán, 2019; Sánchez y García, 2017), entre otros efectos. 
Cabe notar que existe una asociación considerable y significativa entre la calidad de la vida laboral y el compromiso organizacional (Nayak, 2015). El compromiso puede considerarse como un índice sobre el cual se pueden observar o determinar los datos sobre el grado de participación de los empleados en la organización (Omugo, Onuoha y Akhigbe, 2016). Dentro de este marco, el compromiso organizacional se define como el apego a la organización Mowda et al. (1979) citado por Diraviam (2018), esto significa que una persona con alto de compromiso organizacional, se inclina a permanecer en la organización y a trabajar con más esfuerzos para ella.

Atendiendo a estas consideraciones, se puede inferir que, en las organizaciones de educación superior, las autoridades como pilares gerenciales, debe fijar su atención en la calidad de vida en el trabajo de los empleados dentro de los cuales los docentes juegan un importante rol, para obtener una fuerza laboral comprometida incrementando su eficiencia, productividad y competitividad. En este mismo orden y dirección, esta investigación se traza como objetivo, determinar los fundamentos teóricos que permitan diagnosticar el efecto de la calidad de vida laboral sobre el compromiso organizacional en los docentes de las universidades.

\section{REVISIÓN TEÓRICA}

El origen del término "calidad de vida" nace a finales de los años 60 y a principios de los 70 a través del patrocinio de la fundación Ford y el ministerio del trabajo en los Estados Unidos, de una serie de conferencias estimuladas por el entonces ampliamente conocido, fenómeno de la "alienación del trabajador" simbolizado por las huelgas por las condiciones laborales entre la población activa mayoritariamente joven de la nueva planta de monta de la General Motors, de Ohio (Granados, 2011), alcanzando el reconocimiento social e institucional gracias a las actuaciones del "Movimiento de Calidad de vida laboral" (Segurado, y Agulló, 2002). Desde entonces, han sido dos grandes perspectivas sobre las que se conceptualiza el estudio de la calidad de vida laboral, una perspectiva teórico-metodológica, cuyo objetivo es mejorar la calidad de vida del entorno de trabajo en función de los intereses organizacionales y una perspectiva psicológica que destaca la importancia de los aspectos subjetivos del empleado (Davis y Cherns, 1975; Taylor, 1978; Elizur y Shye, 1990; González, Peiró y Bravo, 1996).

Han sido muchas las definiciones sobre calidad de vida laboral que se han ido ofreciendo para ampliar conocimientos en la comunidad científica. En este sentido, la calidad de vida laboral es un proceso para hacer más humano el lugar de trabajo (Walton, 1973), en el que los empleados satisfacen necesidades personales importantes a través de sus experiencias (Suttle, 1977), se establece una relación entre los empleados el trabajo y la organización (Nadler y Lawler, 1983), se comparten los problemas laborales (Delamotte y Takezawa, 1984) y se considera a los empleados como un recurso (Munduate, 1993) de participación en las decisiones (Robbins, 1989) para que puedan gozar de condiciones y ambientes de 
trabajo favorables (Lau, 2000) fundamentadas en el diseño de puestos de trabajo más ergonómicos y en condiciones de trabajo seguras y saludables (Segurado, y Agulló, 2002).

Los paradigmas sobre la calidad de vida laboral (Ahmad, 2013), han sido empíricamente válidos y necesarios para delimitar su campo de investigación (Martel \& Gilles, 2006). Algunos estudios han demostrado el efecto que produce el apoyo del supervisor como factor de calidad de vida laboral en la salud (Dolan, García, Cabezas, y Tzafrir, 2008), otros han comprobado que la ausencia de apoyo y seguridad en el empleo, la remuneración inadecuada, la desigualdad en el trabajo, la falta de reconocimiento y de promoción han traído como consecuencias en las organizaciones una marcada rotación de personal (Mosadeghrad, 2013). Por ello, se hace necesario un enfoque sobre calidad de vida laboral que pueda integrar tanto los intereses individuales como los intereses de la organización para maximizar los beneficios mutuos (Grote \& Guest, 2016).

En el contexto educativo las investigaciones infieren el impacto positivo de la satisfacción laboral con los factores de la calidad de vida laboral de los docentes (Bhavani y Jegadeeshwaran, 2014), el efecto que producen las categorías de la calidad de vida laboral en la motivación para enseñar (Baleghizadeh \& Gordani, 2012). Además, estudios recientes han demostrado que deben mejorarse las condiciones de calidad de vida laboral ya que las percepciones de los docentes sobre el agotamiento y la alienación escolar juegan un papel de mediación parcial en el efecto de sus percepciones sobre la calidad de la vida laboral en el compromiso afectivo (Akar, 2018).

Es pertinente acotar que las universidades son organizaciones que ofrecen servicios de formación profesional en diferentes programas a través de sus recursos, por ello se hace necesario que instauren espacios participativos y dialógicos que faciliten la transformación de un mundo académico rígido a uno participativo, más humano e integral, pues la calidad de vida laboral implica la presencia de múltiples facetas (Acosta, 2016) y las organizaciones con ambiente humanizado permiten que los empleados disfruten de la satisfacción con el trabajo (Donawa, 2018a). No obstante, el aumento en la demanda por recursos para ampliar los niveles de cobertura y mejorar la calidad de los servicios educativos (Melo, Ramos y Hernández, 2017), recientemente han cobrado importancia por el impacto de diferentes variables, como el compromiso organizacional en el desempeño de las instituciones de educación superior. Por ello, se debe distinguir, que el presente estudio se enfoca en la investigación de la percepción de los docentes sobre los factores de la calidad de vida laboral y su efecto sobre el compromiso organizacional desde una perspectiva teórica en contextos universitarios.

En función de las ideas descritas, en términos generales, las investigaciones sobre la calidad de vida laboral en los empleados de educación superior no están satisfechos con sus trabajos y carreras (Edwards, Van Laar, Easton \& Kinman, 2009). En los docentes de las universidades, las investigaciones 
denotan que el trabajo, la satisfacción de la carrera y el equilibrio de casa al trabajo se relacionan negativamente con el compromiso organizacional (Fontinha, Van Laar y Easton, 2016), otros estudios sugieren que las universidades diseñen medidas adecuadas para mejorar el compromiso organizacional, la retención de académicos altamente calificados y reducir la alta rotación de docentes (Subbarayalu \& Al Kuwaiti, 2018).

En otro orden de ideas, es preciso acotar que el compromiso organizacional, es definido paralelamente a la calidad de vida laboral, como un sentimiento de cohesión o participación que surge entre el empleado con una organización (Kanter, 1968). Es un concepto, que adopta un empleado en forma de identificación que consiste en adoptar como propios los objetivos y valores de la organización, en forma de participación que indica la absorción o inmersión psicológica en las actividades del rol de trabajo y en forma de lealtad que nace de un sentimiento de afecto y apego a la organización (Buchanan, 1974). Otras formas de compromiso son el cumplimiento que refleja la participación del comportamiento para recompensas extrínsecas específicas, la identificación basada en un deseo de afiliación con la organización y la internalización en la que un empleado adopta un comportamiento impulsado por valores u objetivos internos que son consistentes con los de la organización (O'Reily y Chatman, 1986). En este sentido, el compromiso es una fuerte creencia y aceptación de los objetivos y valores de la organización, la voluntad de ejercer un esfuerzo considerable en nombre de la organización y un fuerte deseo de mantener la membresía en la organización (Mowday, Porter y Steers, 1982). No obstante, más allá de la categorización del compromiso organizacional, se diseña un modelo que describe el compromiso como un deseo afectivo, una necesidad de continuidad y una obligación normativa (Meyer \& Allen, 1991), que se ha utilizado en numerosos estudios de investigación posteriores y que se explica más adelante.

En la relación del empleado con los factores que determinan la calidad de vida laboral de la organización se determina el compromiso organizacional (Allen \& Meyer, 1990) como una condición psicológica que relaciona los criterios en la relación del empleado en la organización y las implicaciones en la decisión de permanecer en la organización, lo que significa que el empleado comprometido permanecerá en la organización en comparación con el empleado no comprometido.

En las instituciones educativas el compromiso es parte de las reacciones afectivas o emocionales de los docentes ante sus experiencias (Hong, Tan \& Bujang, 2010) y sus conceptualizaciones se vinculan directamente con las investigaciones (Mowday, Steers y Porter, 1979). Las características personales como el estado civil, la edad y el género pueden determinarse en la calidad de la vida laboral y el compromiso organizacional (Chen \& Francesco, 2003). Investigaciones en educación superior muestran el estrés y la presión para publicar y para desarrollar el conocimiento como experto y como investigador (Barlow \& Antoniou, 2007; Worrall-Carter \& Snell, 2003). 
Con base en lo antes indicado, es evidente mencionar que la calidad de vida laboral y el compromiso organizacional juegan un papel determinante en la eficacia de las universidades, por ello, diferentes estudios se han conducido a abordar los factores que se relacionan con la calidad de vida laboral docente subyacentes en la eficiencia de las organizaciones educativas. En este sentido, es pertinente responder a la interrogante sobre la relación que existe entre la percepción de la calidad de vida laboral en los docentes y el compromiso organizacional vinculados al esfuerzo para brindar calidad académica y disminuir la intención de rotación en las universidades.

La situación descrita despierta el interés por identificar a través de estudios recientes, la percepción de los factores que integran la calidad de vida laboral de los docentes y las actitudes hacia el compromiso organizacional en las universidades. En este sentido, la metodología utilizada para el análisis conceptual de las variables se fundamenta en una exhaustiva revisión bibliográfica que se resume a continuación.

\section{Calidad de vida laboral}

La calidad de vida laboral denota todos los aportes de la organización que apuntan a la satisfacción del empleado y a la mejora de la efectividad de la organización, implica desarrollar empleos y condiciones de trabajo que sean excelentes para los empleados, y se refiere al nivel de satisfacción, motivación, compromiso y participación de una experiencia individual con respecto a su línea en el trabajo (Rahiman y Kodikal, 2018). La calidad de vida en el trabajo es la satisfacción general generada por la idoneidad de las tareas, la cultura y el clima organizacional, el espíritu de equipo y de compañerismo, las percepciones salariales y prestaciones, entre otros factores (Meriño, Chirinos, Donawa y Martínez, 2017).

Cabe agregar que, en términos generales, la percepción laboral de un empleado puede definirse como la clasificación, interpretación, análisis e integración de los estímulos que producen impulsos e influyen en su concepción laboral, razón por la cual, la calidad de vida laboral se define, como la percepción de los impulsos motivacionales que tiene un empleado sobre las condiciones que causan satisfacción en el contexto laboral dentro de la organización para la cual presta sus servicios (Donawa, 2018b). Estas condiciones, eventos o factores que causan satisfacción laboral son los parámetros en los que se sustenta la calidad de vida laboral. Estudios recientes (Subbarayalu \& Al Kuwaiti, 2018) luego de considerar los modelos de calidad de vida laboral establecieron cinco componentes, identificadas en esta investigación como dimensiones, para medir la calidad de vida laboral en los docentes de las universidades:

\section{Dimensiones de la Calidad de Vida Laboral.}

\section{Las condiciones de trabajo.}


Estas condiciones se refieren a una justa asignación de responsabilidades en función de la capacidad, contar con los recursos para el desempeño de las actividades, apoyo para realizar investigaciones y la transparencia en la toma de decisiones.

\section{Los factores psicosociales en el lugar de trabajo.}

Esta dimensión de la calidad de vida laboral hace referencia a la posibilidad del empleado de expresar sus opiniones en el área de trabajo, sentirse presionado por el tiempo en las tareas asignadas, recibir apoyo de los colegas y lograr el equilibrio saludable entre el trabajo y la vida en el hogar.

\section{Las oportunidades de formación y desarrollo.}

Son las oportunidades de apoyo que brinda la universidad para participar en programas de capacitación, talleres de desarrollo académico y profesional.

\section{La compensación y las recompensas.}

Se refiere contar con las condiciones salariales justas, transparencia en para las promociones, las compensaciones y otros beneficios complementarios.

\section{La satisfacción laboral y la seguridad laboral.}

Son las condiciones que le brinda la universidad sobre la estabilidad laboral, planificar el horario, usar conocimientos para desarrollar habilidades, gozar de autoridad y responsabilidad como docente.

Los componentes de la calidad de la vida laboral en el sector de la educación superior tienen implicaciones importantes para el compromiso, significa que cuando el ambiente y las condiciones de trabajo son positivas, hay satisfacción laboral y compromiso de los empleados (Ramasamy y Renganathan, 2017).

\section{Compromiso organizacional.}

En la actualidad, las organizaciones deben enfrentar una presión competitiva cada vez mayor, para sobrevivir y aumentar la competitividad de las instituciones, uno de los conceptos de gestión desarrollados es el compromiso organizacional (Martínez, Vega y Eternod, 2017) que ha captado la atención de los investigadores. Los autores del compromiso organizacional (Allen \& Meyer, 1990) lo definen como la intención de los empleados de permanecer en la organización, señalan que el compromiso tiene tres estados psicológicos, el compromiso afectivo, de continuidad y normativo. Enuncian que se ven mejor como componentes distinguibles, en lugar de tipos, de compromiso actitudinal; es decir, los empleados pueden experimentar cada uno de estos estados psicológicos en diversos grados. En este sentido acotan que el compromiso se compone de tres dimensiones que incluyen: 


\section{Compromiso afectivo.}

El compromiso afectivo es el enfoque más prevalente del compromiso organizacional en la literatura es aquel en el que el compromiso se considera un vínculo afectivo o emocional con la organización, de manera que la persona fuertemente comprometida se identifica, participa y disfruta de la membresía en la organización. Esta dimensión se refiere al vínculo emocional del empleado, la identidad y la participación en los asuntos de la organización, describe una orientación afectiva que siente el empleado hacia la organización por lo que es aquella fuerza interna, que identifica al individuo con la organización. Un empleado con un alto compromiso afectivo está vinculado a una organización porque quiere serlo (Zaraket, 2018).

\section{Compromiso de continuidad.}

Se refiere al conocimiento del costo de abandonar la organización, implica que los empleados con vínculo principal de organización debido al compromiso de continuidad permanecen en la organización ya que necesitan este trabajo, la mayoría de las personas están obligadas a continuar trabajando, ya que no quieren perderlo ya que no hay alternativa. Un empleado con un alto nivel de compromiso de continuidad es leal al empleador porque debe serlo (Zaraket, 2018).

\section{Compromiso normativo.}

Esta dimensión se centra en los sentimientos de lealtad a una organización particular como resultado de la internalización de las presiones normativas ejercidas sobre un individuo. Los empleados exhiben comportamientos comprometidos porque es lo correcto, los empleados que tienen un compromiso normativo sienten que deben permanecer en la organización. Los empleados con compromiso normativo están convencidos de que deben permanecer en una organización porque, desde su consideración, es "moralmente lo correcto" (Zaraket, 2018).

La situación descrita, despierta el interés por realizar una investigación sobre la percepción de los factores que afectan la calidad de vida laboral y su relación con el compromiso organizacional en los docentes de las universidades. La relación de los docentes con los factores que determinan la calidad de vida laboral de las universidades determina el compromiso organizacional como una condición psicológica que relaciona los criterios del entorno laboral y las implicaciones en la decisión de permanecer en ella, lo que significa que el docente comprometido permanecerá en la organización en comparación con el docente no comprometido.

\section{CONCLUSIONES}


Las universidades se desarrollan en un entorno altamente competitivo y globalizado donde el factor humano, independientemente de los avances tecnológicos, sigue siendo el factor clave para alcanzar los objetivos estratégicos, dichos objetivos nacen de la razón de ser de la organización y su cumplimiento requerirá de la participación de sus docentes como empleados, esta participación podría ser más acertada si el docente logra una condición adecuada del entorno de trabajo, donde tanto sus necesidades laborales como profesionales logren un equilibrio con las necesidades institucionales para obtener el compromiso organizacional.

Dentro de esta perspectiva, las dimensiones de la calidad de vida laboral que están representadas por las condiciones de trabajo, los factores psicosociales en el lugar de trabajo, las oportunidades de formación y desarrollo, la compensación y las recompensas y la satisfacción laboral y la seguridad laboral; representan los componentes clave para medir la percepción de los factores que causan satisfacción laboral en los docentes universitarios. Del mismo modo, las dimensiones del compromiso organizacional, distinguidas en tres formas de compromiso que son el afectivo, el de continuidad y el normativo; permiten medir la percepción del estado psicológico del docente para su permanencia en la universidad.

Los enfoques teóricos reflexionar sobre la importancia de las variables y permiten inferir que, cuando la percepción de los factores de la calidad de vida en los docentes produce como resultado satisfacción laboral, esta respuesta causaría un efecto significativamente positivo sobre el compromiso organizacional. A partir de la descomposición teórica de estas dos variables, las investigaciones futuras podrán construir un instrumento de recolección de datos diseñando afirmaciones o preguntas que indiquen comportamientos actitudinales sobre la percepción de la calidad de vida laboral en los docentes de las universidades y su efecto sobre el compromiso organizacional.

\section{REFERENCIAS BILBIOGRÁFICAS}

Acosta, R. (2016). Conceptos de calidad de vida laboral en el ámbito docente universitario. Revista Salud Bosque, 5(2), pp. 89-100.

Allen, N. J., \& Meyer, J. P. (1990). The measurement and antecedents of affective, continuance and normative commitment to the organization. Journal of Occupational Psychology, 63(1), pp. 1-18.

Baleghizadeh, S., \& Gordani, Y. (2012). Motivation and quality of work life among secondary school efl teachers. Australian Journal of Teacher Education , 37(7), pp. 30-42

Barlow, J. \& Antoniou, M. (2007). Room for improvement: The experiences of new lecturers in higher education. Innovations in Education \&Teaching International, 44(1), pp. 67-77.

Chen, Z. \& Francesco, A. (2003). The relationship between the three components of commitment and employee performance in China. Journal of Vocational Behavior, 62(3), pp. 490-510. 
Cruz, J. (2018). La calidad de vida laboral y el estudio del recurso humano: una reflexión sobre su relación con las variables organizacionales. Pensamiento \& gestión, 45, pp. 58-81.

Delgado, S., López, V. y Moreno, L. (2017). Impacto del Clima Organizacional en la Rotación del Personal: Evidencia en Sector Desarrollo de Software. Revista Internacional Administración y Finanzas, 10(1), pp. 49-62.

Diraviam, A. (2018). Un estudio sobre la relación entre las cualidades de la vida laboral en el compromiso organizacional entre profesionales de la salud. Journal of Business and Management, 18(9), pp. 76-85.

Donawa, Z. (2018a). Gestión gerencial para un ambiente más humano en las organizaciones. Novun, 8(1), pp. 144-163.

Donawa, Z. (2018b). Percepción de la calidad de vida laboral en los empleados en las organizaciones. Novun, 2(8), pp.43-63.

Edwards, J., Van Laar, D., Easton, S., \& Kinman, G. (2009). The Work-related Quality of Life Scale for Higher Education Employees. Quality in Higher Education, 15(3), pp. 207-219.

Fontinha, R., Van Laar, D., \& Easton, S. (2016). Quality of working life of academics and researchers in the UK: the roles of contract type, tenure and university ranking. Studies in Higher Education, 43(4), pp. 786-806.

Granados, I. (2011). Calidad de vida laboral: Historia, dimensiones y beneficios. Revista IIPS, 14(2), pp. $271-276$.

Grote, G., \& Guest, D. (2016). The case for reinvigorating quality of working life research . Human Relations, pp., 1-19.

Hong, K., Tan, K., \& Bujang, S. (2010). Relationships between work life quality of teachers with work commitment, stress and satisfaction: A study In Kuching, Sarawak, Malaysia. Jurnal Teknologi, 52, pp. 1-15.

Kanter, R. (1968). El compromiso y la organización social: Un estudio de compromiso mecanismos en las comunidades utópicas. American Sociological Review, 33, 499-517.

Martel, J. \& Gilles, D. (2006). Quality of Work Life: Theoretical and Methodological Problems, and Presentation of New Model and Measuring Instrument. Social Indicators Research 77, pp. 333368.

Martínez, M., Vega J. y Eternod, V. (2017). La influencia del compromiso organizacional y orientación al aprendizaje sobre la innovación en las pymes. Contaduría y Administración, 63 (3), pp. 1-19

Matabanchoy, S., Álvarez, K. y Riobamba, O. (2019). Efectos de la evaluación de desempeño en la calidad de vida laboral del trabajador: revisión del tema entre 2008-2018. Universidad y Salud, $21(2)$, pp. 176-187. 
Melo-Becerra, L., Ramos-Forero, J. y Hernández-Santamaría, P. (2017). La educación superior en Colombia: situación actual y análisis de eficiencia. Revista Desarrollo y Sociedad, pp. 59-111.

Meriño, V., Chirinos Y., Donawa, Z. y Martínez, R. (2017). Calidad de vida laboral en el personal administrativo de la Universidad Nacional Experimental Rafael María Baralt. En Meriño, V., Chirinos Y., Camejo L. y Martínez C. Gestión del Conocimiento. Perspectiva Multidisciplinaria, Volumen III, pp. 2-25. Colección Unión Global. Venezuela: Fondo Editorial Universitario Servando Garcés de la Universidad Politécnica Territorial de Falcón Alonso Gamero.

Mowday, R., Porter, L. y Steers, R. (1982). Vínculos Empleado-organización: La psicología de compromiso, el absentismo y la rotación. Nueva York: Academic Press.

Mowday, R., Steers, R. y Porter, L. (1979). The measurement of organizational commitment. Journal of Vocational Behavior, 14(2), pp. 224-247.

Nayak, T. (2015). Calidad de vida laboral y desempeño organizacional, el papel mediador del compromiso de los empleados. Journal of Health Management, 17(3) pp. 263-273.

O'Reilly, C. \& Chatman, J. (1986). Organizational Commitment and Psychological Attachment: The effects of Compliance, Identification and Internalization on Pro-social Behaviour. Journal of Applied Psychology, 1986, 6 (71), pp. 492-499.

Omugo, O., Onuoha, B. y Akhigbe, J. (2016). Calidad de vida laboral y compromiso organizacional en organizaciones del sector público en el estado de Rivers. International Journal of Novel Research in Humanity and Social Sciences, 3(3), pp. 35-44.

Rahiman, H. y Kodikal, R. (2018). Calidad de vida laboral: una revisión empírica. Research journal of commerce \& behavioural science, 7(7), pp. 1-10.

Ramasamy, A. y Renganathan, B. (2017). Calidad de vida laboral en el sector de la educación superior: hacia una perspectiva integrada. International Journal of Management, 8(1), pp. 62-72.

Sánchez, M. y García, M. (2017). Satisfacción Laboral en los Entornos de Trabajo. Una exploración cualitativa para su estudio. Scientia Et Technica, 22(2), pp. 161-166.

Segurado, A. y Agulló, E. (2002). Calidad de vida laboral: hacia un enfoque integrador desde la Psicología Social. Psicothema, 14(4), pp. 828-836.

Subbarayalu, A. \& Al Kuwaiti, A. (2018). Quality of Work Life of Teaching Staff Working at a Higher Education Institution in Saudi Arabia: A Cross-sectional Study. Journal of Social Service Research, DOI: 10.1080/01488376.2018.1481173.

Tatamuez-Tarapues, R., Domínguez, A. y Matabanchoy-Tulcán, S. (2019). Revisión sistemática: Factores asociados al ausentismo laboral en países de América Latina. Universidad y Salud, 21(1), pp. 100-112.

Worrall-Carter, L., \& Snell, R. (2003). Nurse academics meeting the challenges of scholarship and 
research. Contemporary Nurse, 16(1-2), pp. 40-50.

Zaraket, W. (2018). La influencia del compromiso organizacional en la construcción de marca organizacional. International Journal of Economics, Commerce and Management, 6(2), pp. 399416. 